\title{
Behavioral Disassociation of Perceived Sweet Taste Intensity and Hedonically Positive Palatability
}

\author{
[DEsmeralda Fonseca, ${ }^{1}$ Vicente Sandoval-Herrera, ${ }^{1,2}$-Sidney A. Simon, ${ }^{3}$ and ${ }^{\circ}$ Ranier Gutierrez ${ }^{1}$
}

https://doi.org/10.1523/ENEURO.0268-20.2020

${ }^{1}$ Department of Pharmacology, CINVESTAV, Laboratory of Neurobiology of Appetite, Mexico City 07360, Mexico, ${ }^{2}$ Departamento de Fisiología, Escuela Nacional de Ciencias Biológicas, Instituto Politécnico Nacional, Wilfrido Massieu, Mexico City 07738, México, and ${ }^{3}$ Department of Neurobiology, Duke University Medical Center, Durham, NC 27710

\begin{abstract}
The intensity of sucrose (its perceived concentration) and its palatability (positive hedonic valence associated with ingestion) are two taste attributes that increase its attractiveness and overconsumption. Although both sensory attributes covary, in that increases in sucrose concentration leads to similar increases in its palatability, this covariation does not imply that they are part of the same process or whether they represent separate processes. Both these possibilities are considered in the literature. For this reason, we tested whether sucrose's perceived intensity could be separated from its hedonically positive palatability. To address this issue, rats were trained in a sucrose intensity task to report the perceived intensity of a range of sucrose concentrations before and after its palatability was changed using a conditioned taste aversion (CTA) protocol. We found that the subjects' performance remained essentially unchanged, although its palatability was changed from hedonically positive to negative. Overall, these data demonstrate that sucrose's perceived intensity and its positive palatability can be dissociated, meaning that changes of one taste attribute render the other mostly unaffected. Thus, the intensity attribute is sufficient to inform the perceptual judgments of sucrose's concentrations.
\end{abstract}

Key words: intensity; palatability; sucrose; sweetness; taste quality

\section{Significance Statement}

Subjects trained to classify sucrose concentrations could guide their decisions based on their perceived intensity and/or palatability. Despite the fact that both taste attributes covary with changes in sucrose concentration, we found that it was possible to change its hedonically positive palatability attribute without affecting the sensitivity to identify sucrose's perceived intensity. That is, the subjects perceptual judgments of sucrose concentrations were based on the information provided by the intensity attribute showing, for the first time, that the hedonically positive aspect of sucrose's palatability and its perceived intensity could be dissociated.

\section{Introduction}

Mammals, including rodents and humans, have the impressive ability to identify and consume sweet-tasting, energy possessing, molecules, such as sucrose, and to avoid bitter-tasting molecules they find aversive. The consumption of sweet-tasting molecules to humans generates a taste percept that consists of a stable component,

Received June 18, 2020; accepted September 11, 2020; First published October 19, 2020

The authors declare no competing financial interests. the tastant quality, which is presumed to remain constant (Staszko et al., 2020), and a variable component, its palatability (or hedonic value), whose value is dependent on a variety of factors including its internal states and learning experiences (Grill and Norgren, 1978a; Garcia et al., 1985). It is the palatability component that elicits the activation of oromotor responses that result in the consumption or rejection of foods

Author contributions: E.F., S.A.S., and R.G. designed research; E.F. and V.S.-H. performed research; E.F. analyzed data; E.F., S.A.S., and R.G. wrote the paper. 
(Grill and Norgren, 1978b; Berridge and Grill, 1983). One tried and tested way to change a tastant's palatability is through a conditioned taste aversion (CTA), where a tastant is paired with an unpleasant agent (Grill and Norgren, 1978a). In virtually all of these CTA experiments, a single tastant concentration is tested (Domjan, 1975; Ninomiya et al., 1984; Kotlus and Blizard, 1998) and, although for sucrose, it is assumed the quality (identity) remains unchanged with changes in concentration (see above), the perceived intensity has not been dissociated.

In rodents, the palatability of sucrose has been measured using a brief access test by counting the number of sucrose-evoked licks in a small time window (Davis, 1973; Spector and Smith, 1984; Spector et al., 1998; Dotson and Spector, 2004), or using a taste reactivity test to record orofacial movements, such as gaping and tongue protrusions (Grill and Norgren, 1978b,c; St. John and Spector, 2008; Berridge et al., 2009). As noted, both oromotor palatability responses and the perceived intensity increase as the concentration of sucrose increases. However, the perceived intensity attribute cannot be directly measured by licking responses per se because it does not give information about the subject's perception. For this reason, taste intensity discrimination tasks are better suited to obtain a judgment report of the perceived intensity of a tastant (Colbert et al., 2004; MacDonald et al., 2012; Fonseca et al., 2018). Here, we performed behavioral studies to determine whether the perceived intensity of sucrose is affected by giving a CTA that causes its palatability to be shifted from positive to negative.

In the rodent primary gustatory cortex (GC), electrophysiological recordings revealed competing viewpoints regarding how quality and palatability are encoded. Some studies, in which usually single concentrations of tastants were delivered to the animals, either passively (via an intraoral cannula) or actively (head-fixed licking), revealed that the processing of taste quality and palatability occurs serially with quality occurring first (Katz et al., 2001; Jezzini et al., 2013; Levitan et al., 2019). Using optogenetic manipulations, other studies found that tastant identity and palatability are processed by distinct channels or circuits (Wang et al., 2018). Specifically, Wang et al. (2018) were able to reverse the hedonic value of a sweet or bitter tastant by stimulating distinct insular cortex (IC) projections to the amygdala. They showed that mice with silenced neurons in the amygdala no longer exhibited oromotor palatability responses usually evoked by sweettasting or bitter-tasting chemicals, as perceived by

This work was supported in part by the Productos Medix Grant 3247, Cátedra Marcos Moshinsky, and Fundación Miguel Alemán and CONACyT Fronteras de la Ciencia Grant 63, Problemas Nacionales 462 (to R.G.).

Acknowledgements: We thank Fabiola Hernandez for their invaluable support on animal care.

Correspondence should be addressed to Ranier Gutierrez at ranier@ cinvestav.mx.

https://doi.org/10.1523/ENEURO.0268-20.2020

Copyright (C) 2020 Fonseca et al.

This is an open-access article distributed under the terms of the Creative Commons Attribution 4.0 International license, which permits unrestricted use, distribution and reproduction in any medium provided that the original work is properly attributed. humans. Interestingly, this occurs without affecting the ability of the same mice to recognize the quality of these tastants, thus suggesting that palatability and taste quality are two separate processes that can be dissociated (Wang et al., 2018). Of course, different anterior-posterior regions of the IC and their specific projections to the amygdala (and other regions) could also play a broader role in processing emotional states, such as pleasure and disgust-related facial expressions, beyond just conveying pure taste information (Dolensek et al., 2020). However, whichever the taste coding process proves to be correct, it is currently unknown whether palatability and the perceived intensity of sucrose solutions could be dissociated, and to what extent the removal of one orosensory attribute would impact the other.

Our goal was to determine whether the perceived intensity of sucrose could be behaviorally disentangled from its hedonically positive palatability attribute and, if it could, then determine its impact on the animal's sensitivity to identify sucrose concentrations. This was accomplished using a novel behavioral approach that combined a sucrose concentration discrimination-generalization task to evaluate its perceived intensity and a CTA protocol to change its palatability from positive to negative. In sum, we found that the perceived intensity attribute was sufficient to inform the subjects about the sucrose concentration, indicating that the hedonic evaluation of a taste stimulus is dissociable from its intensity-discriminative properties.

\section{Materials and Methods}

\section{Animals}

We used nine male Sprague Dawley rats weighing 300$320 \mathrm{~g}$. Animals were individually housed in standard laboratory cages in a temperature-controlled $\left(22 \pm 1^{\circ} \mathrm{C}\right)$ room with a 12/12 h light/dark cycle (lights were on 7 A.M. and off at 7 P.M.). All procedures were approved by the Institutional Animal Care and Use Committee. Rats were given ad libitum access to Chow food (PicoLab Rodent Diet 20) in their home cage. Water was available for $30 \mathrm{~min}$ after discrimination/generalization sessions, 4560 min during CTA (see below for details), and $24 \mathrm{~h}$ during the two-bottle preference test. All experiments were performed from 1 to 4 P.M., since we found rats more alert and motivated to work during this period.

\section{Sapid stimuli}

Sucrose was reagent-grade chemical quality purchased from Sigma-Aldrich (Mexico). It was dissolved in distilled water, and the following concentrations were used: 3, 4.75, 7.5, 11.75, and 18wt/vol\% (Fonseca et al., 2018). Solutions were freshly prepared every other day and maintained under refrigeration. Solutions were used at room temperature.

\section{Behavioral equipment}

Animals were trained in four identical standard operant conditioning chambers of internal dimensions $30.5 \times$ $24.1 \times 21.0 \mathrm{~cm}$ (Med Associates Inc.; Fonseca et al., 
2018). The front panel of each chamber was equipped with one central and two lateral V-shape licking ports with a photobeam sensor to register individual licks (Med Associates Inc.). Each port had a licking spout that consisted of either one (for lateral ports) or a bundle of up to six (for the central port) blunted needles (20-gauge) that were carefully sanded and glued at the tip of a stainlesssteel sipper tube. Each needle was connected to a solenoid valve (Parker) via a silicon tube. The drop volume was calibrated before each session and maintained by using an individual and constant air pressure system (Fonseca et al., 2018). On the rear panel, there was an ambiance masking noise amplifier with a speaker that was turned on during the entire sessions to reduce external sounds from valves opening. A light was located on the front wall of the box. Chambers were enclosed in a ventilated, sound-attenuating cubicle. Experimental events were controlled and registered by a computer via a Med Associates interface (Med Associates Inc.).

\section{Sucrose discrimination and generalization protocols}

During discrimination sessions, the subjects were trained to produce differential responses based on the concentration of one drop of sucrose: to go to the left if low sucrose (3\%) was received and go to the right if it was high (18\%). These conditions were counterbalanced across subjects. The task comprised five epochs: return, pre-cue, cue-dependent licks (CDL), response, and outcome (also see Fonseca et al., 2018). The lights were on, and a trial began when an animal moved from the lateral port to the central port and emitted one dry lick (return). After two to three dry licks (pre-cue), the subject received a $10-\mu l$ drop of sucrose solution. Subsequent licks were dry and had no programmed consequences. The time spent licking in the central port from cue delivery onset comprised the CDLs epoch. Next, after sampling the cue, the subjects had to move from the central to a lateral port and emit one dry lick. If the animal chose the correct port (i.e., left $\rightarrow$ low, right $\rightarrow$ high), it received three 10- $\mu$ l drops of water; otherwise, the lights went off for $50 \mathrm{~ms}$. There was no fixed time limit to emit a response; although to initiate a new trial, a single lick in either port was required. Once the animals reached the learning criterion, defined as four consecutive sessions with $\geq 75 \%$ correct responses, generalization sessions were introduced. In these sessions, $80 \%$ of the trials were discrimination trials, which were identical to the trials during discrimination sessions; the remaining were generalization trials, in which one of five sucrose concentrations $(3 \%, 4.75 \%$, $7.5 \%, 11.75 \%$, and $18 \%$ ) or water $(0 \%)$ were delivered, and the animals had to report whether they perceived it as low or high. The perceived intensity was defined as the percentage of responses emitted in the high-sucrose spout. In order to avoid any perceptual bias, no responses during generalization trials were rewarded (Fonseca et al., 2018). Once the response was emitted, the lights went off for $50 \mathrm{~ms}$. Discrimination and generalization trials were pseudo-randomly interleaved, such that discrimination trials always flanked one generalization trial; the same was true for generalization sessions: they were preceded and followed by discrimination sessions. Flanking generalization trials and sessions, by discrimination trials and sessions, respectively, ensured a stable stimulus control intra and between sessions, as seen in the organized increase of high-sucrose responses as a function of sucrose concentration. Both discrimination and generalization trials lasted $20 \mathrm{~min}$ independently of the number of trials completed. The subjects had $30 \mathrm{~min}$ of access to water after discrimination and generalization sessions.

\section{CTA}

Once the animals learned to guide their choices using the sucrose concentration as a cue, they underwent a CTA protocol to shift sucrose's positive hedonic value into a negative one. Initially, over three consecutive days, the animals had 1-h access to water (baseline). The following day, they had access to one bottle filled with sucrose $18 \%$, and after $15 \mathrm{~min}$, they received an intraperitoneal injection $7.5 \mathrm{ml} / \mathrm{kg}$ of $0.2 \mathrm{M} \mathrm{LiCl}$ and then had access to water for $45 \mathrm{~min}$ (CTA-1). The animals were then allowed to recover for $1 \mathrm{~d}$ and had $1-\mathrm{h}$ access to water. The following day, they went through a test $1+$ CTA-2, where they were allowed to drink sucrose $18 \%$ for $15 \mathrm{~min}$ followed by a second intraperitoneal $\mathrm{LiCl}$ injection and then had 45 -min access to water period. Animals were allowed to recover for the next 24 $\mathrm{h}$ with access to the water for $1 \mathrm{~h}$. Finally, on the next day, the animals received 15 min of access to $18 \%$ sucrose, followed by $45 \mathrm{~min}$ of access to water (test 2). After they were given two CTAs, the animals were given $3 \mathrm{~d}$ of free access to water; afterward, their performance was re-evaluated in the sucrose intensity task following the same protocol described above.

\section{Two-bottle preference test}

A two-bottle preference test was performed to confirm that sucrose maintained a negative valence after re-evaluation in the sucrose intensity task. Briefly, the subjects had three baseline days with ad libitum access to water. In the next $2 \mathrm{~d}$, they had $24 \mathrm{~h}$ of access to two bottles, one with sucrose $18 \%$ and the other with water, and each bottle was available on each side of their home cage. The bottle positions were counterbalanced across days.

\section{Data analysis}

All data analysis was performed using MATLAB 2019b (The MathWorks Inc.) and GraphPad Prism 8. Data obtained from discrimination and generalization sessions are presented as mean \pm SEM and were treated with parametric statistics. To compare the impact of the CTA in the discrimination and generalization measurements, the data from the five discrimination/generalization sessions before CTA were concatenated and compared with the concatenated data from the five sessions after CTA. Differences among the data mentioned above were identified using parametric statistics. Data obtained during the CTA protocol and the two-bottle test are presented as median \pm interquartile range (iqr) and were treated with non-parametric statistics, since only one observation per subject was obtained, and the size of the groups was relatively small $\left(n_{\text {saline }}=4, n_{\mathrm{LiCl}}=5\right)$. Unless otherwise indicated, $\alpha$ level was set up at 0.05 . All statistical results can be found in the Extended Data Table 1-1. 


\section{Sucrose intensity task: discrimination and generalization}

To identify whether there was a significant difference in performance across sessions, a mixed-effects model was employed (Bagiella et al., 2000). To identify when the performance started to be significantly different from the first day, we used Dunnett's multiple comparisons test (Dunnett, 1955). Changes in CDL, response, return, outcome differences by session, concentration (low vs high), or interaction (session $\times$ concentration) were assessed by a mixed-effects model (Bagiella et al., 2000). The same approach was applied to licking given in the outcome epoch divided by rewarded versus unrewarded trials instead of concentration. The generalization curves were obtained by quantifying the number of responses emitted to the port associated with high sucrose for each sucrose concentrations given during the generalization trials and fitting the following four-parameter sigmoidal function to those data points:

$$
\mathrm{P}(\text { High })=y_{0}+\frac{a}{1+\mathrm{e}^{((x o-x) * b)}}
$$

where $y_{o}$ is the left endpoint, $a$ is the asymptote (minimum and maximum high-sucrose responses), $x_{O}$ represents the point of the inflection of the curve, $x$ is the $\log _{10}$ sucrose concentrations, and $b$ is the slope. The psychophysical measurements were obtained from the fitted curve: point of subjective equality (PSE) is the concentration that elicits $50 \%$ of high-sucrose responses, that is, the concentration that is equally likely to be perceived as "low" or "high." Limen (Li) is defined as the difference between the concentrations that elicited $75 \%$ and $25 \%$ of high-sucrose responses divided by 2 , indicates the amount of concentration that needs to be changed to perceive that a change in the stimulus has occurred. Finally, the Weber fraction (WF), which is a sensitivity measure, was computed as from the division of the Li over the PSE. In contrast to the just noticeable differences (JNDs) protocol, where only one standard concentration is used (Colbert et al., 2004), in our task, when the rats are presented with the test-stimuli (a drop of sucrose), they then need to report the similarity of the test stimulus to either the lower $(3 \%)$ or the higher $(18 \%)$ standard concentration tested. This approach is similar to what has been employed in temporal bisection tasks (Church and Deluty, 1977).

Differences in the sucrose-high classification responses from the generalization sessions were analyzed using a one-way ANOVA, followed by Dunnett's multiple comparisons test using responses during the $3 \%$ discrimination trials as control.

\section{CTA}

To confirm significant differences in the amount of liquid consumed during the first $15 \mathrm{~min}$ of liquid access, a Mann-Whitney's U test was performed (Kerby, 2014).

\section{Sucrose intensity task re-evaluation}

To identify differences in the performance, CDL, response time, return time, and the number of trials because of sucrose devaluation, the value of these behavioral measurements during the five pre-CTA versus the five post-CTA discrimination sessions were compared using a Student's $t$ test. The same approach was used to detect significant changes in the PSE, Li, and WF during the five generalization sessions pre-CTA versus postCTA. Sucrose-high classification responses in each group (saline or $\mathrm{LiCl}$ ) was compared before and after the treatment by a two-way ANOVA.

\section{Two-bottle preference test}

The preference ratio (PR) was defined as the sucrose intake divided by the total (water + sucrose) intake during a 24-h period. The differences in the PR between saline and $\mathrm{LiCl}$ groups were tested using Mann-Whitney's $U$ test.

\section{Results}

In the sucrose intensity task, rats were trained to lick a central spout and then report whether they perceived 3\% versus $18 \%$ sucrose concentration either as "low" or "high" by responding on the left or right port, respectively (Fig. 1A,B). Briefly, the task was divided into five epochs: return, where the animals return from lateral to the central port to initiate a trial; pre-cue (dry licks gave before cue delivery); CDL, where the animals emitted the dry licks evoked by cue delivery; response, where the animals moved from the central to a lateral port to collect the reward; and outcome, where animals were given a correct response, licked a lateral spout to obtained three drops of water as a reward. After learning this intensity task, we introduced generalization sessions, where the animals were now required to generalize their responses to multiple sucrose concentrations between $3 \%$ and $18 \%$ sucrose (Fig. 1C).

\section{Acquisition of the sucrose intensity task}

In the intensity task the animal's performance increased until they achieved $\geq 75 \%$ correct responses after $19 \pm 4$ sessions (Fig. 2A, the learning criterion, purple line). Dunnett's multiple comparison test showed that compared with day 1 , significant differences were achieved after day $7(p=0.01$; Fig. $2 A$, arrow). The CDLs time in seconds, which is a measure of sucrose's palatability (Perez et al., 2013), decreased across sessions $\left(F_{(40,606)}=\right.$ 6.8, $p<0.0001$; Extended Data Fig. 2-1A). However, there were no significant effects of the concentration factor $\left(F_{(1,16)}=0.02, p>0.05\right)$ or interaction sessions $\times$ concentration $\left(F_{(40,606)}=0.6, p>0.05\right)$. The fact that CDLs were not modulated by sucrose concentrations could reflect a masking effect on palatability responses since sucrose also serves as a discriminative cue that directs operant responding motivated by water deprivation and water reinforcement. Likewise, the response movement (sessions $F_{(40,606)}=8.2, p<0.0001$; Extended Data Fig. $2-1 B$ ) and the return movement (sessions $F_{(40,606)}=10.8$, $p<0.0001$; Extended Data Fig. 2-1C) movement times significantly decreased early in training (from session 2 to 6). However, late in training, they increased and remained at the same level across sessions. Neither the concentration factor (response: $F_{(1,16)}=0.03, p>0.05$; return: $\left.F_{(1,16)}=0.7, p>0.05\right)$, nor a significant effect for interaction between factors were significant (response: $F_{(40,606)}=$ $0.5, p>0.05$; return: $\left.F_{(40,606)}=0.5, p>0.05\right)$. In sum, in this sucrose intensity task, neither CDLs, response, or the 


\section{A}
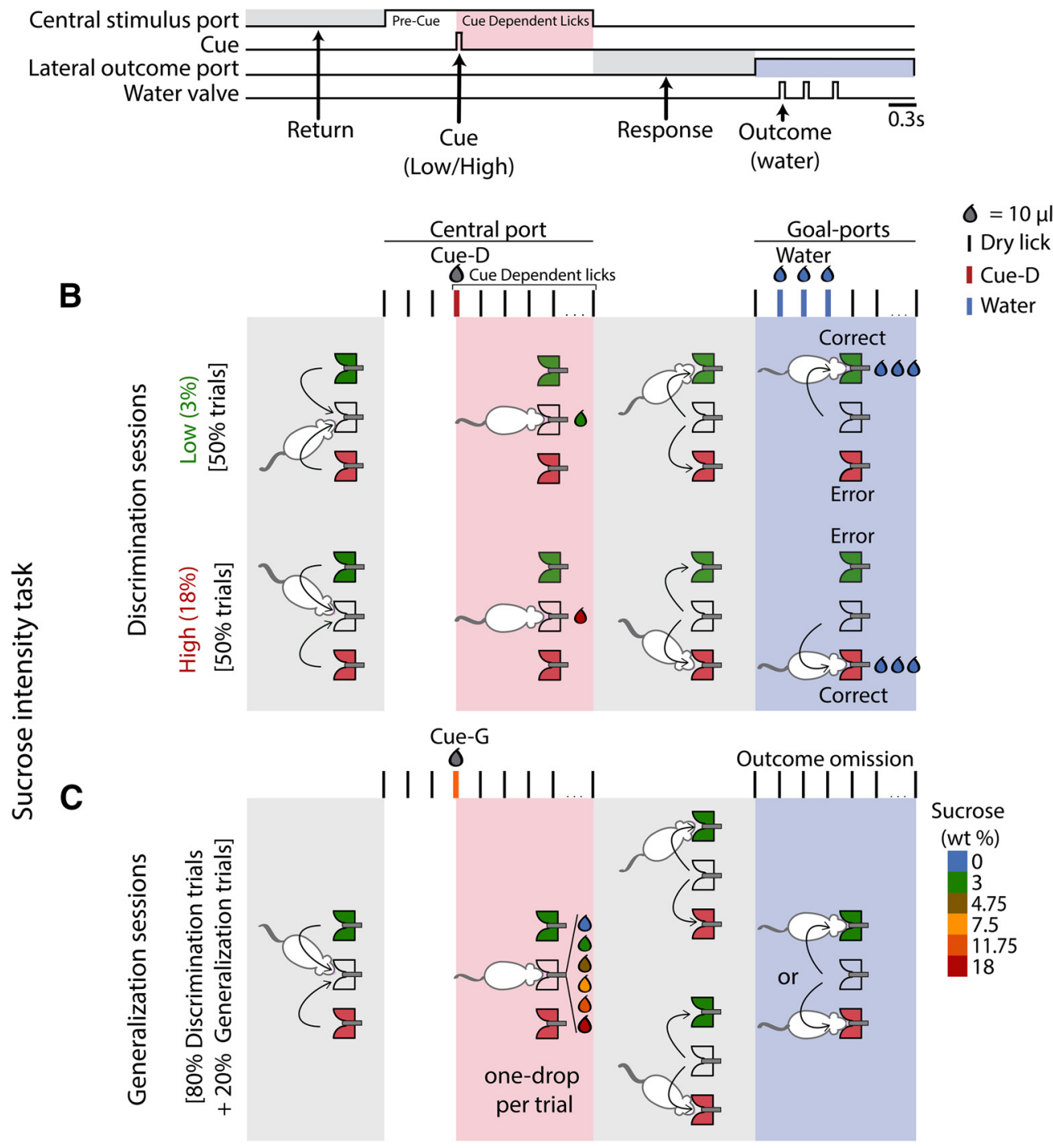

Figure 1. Structure of the sucrose intensity task. $\boldsymbol{A}, \mathrm{A}$ schematic of the task that is comprised of discrimination $(\boldsymbol{B})$ and generalization $(\boldsymbol{C})$ sessions. $\boldsymbol{B}$, During discrimination trials, the subjects moved toward the central port (return epoch) to lick and receive a single $10-\mu$ drop of either a $3 \%$ sucrose-low (green) or 18\% sucrose-high (red). The licks emitted in the central port comprise the cue epoch. The animals had to emit three dry licks before receiving the cue (pre-cue) and then produce CDL before detecting whether the concentration was high or low by going to the left side for a low concentration or to the right side for a high concentration (response epoch), counterbalanced across subjects. For making a correct response, and after one dry lick in the chosen lateral port, they would receive three drops of water. For incorrect choices, the trial was ended (outcome epoch). $\boldsymbol{C}$, Generalization sessions were composed of $80 \%$ discrimination trials (cue-D) and $20 \%$ generalization trials (cue-G). The trial structure of generalization trials is the same as in discrimination, but with two main differences: (1) the subjects received one drop of either six sucrose concentrations $(0 \%, 3 \%, 4.75 \%, 7.5 \%, 11.75 \%, 18 \%)$; and (2) no reward was given during the outcome epoch.

return movement was dependent on the sucrose concentration.

With regard to the licks in the outcome epoch, there was a significant difference between rewarded and unrewarded trials (factor trial type; $F_{(1,16)}=394.9, p<0.0001$; Fig. $2 B$ ), but no differences among concentrations were found $\left(F_{(1,16)}\right.$ $=0.02, p>0.05$; Extended Data Fig. 2-1D). Thus, subjects were highly sensitive to the outcome of the task. Rats detected the absence of reward in $<300 \mathrm{~ms}$ (Fig. $2 B$, black circles; Fonseca et al., 2018). Furthermore, licking during both rewarded and unrewarded trials significantly decreased as a function of sessions $\left(F_{(40,606)}=3.85, p<\right.$ $0.0001)$. Thus, rats reduce the time spent doing unnecessary licking, which they could use to increase the number of trials, thereby increasing the amount of water reward obtained per session.

\section{Generalization trials}

Once the animals learned to discriminate a low (3\%) from a high (18\%) sucrose concentration, the generalization trials began with the discrimination trials interleaved with them 

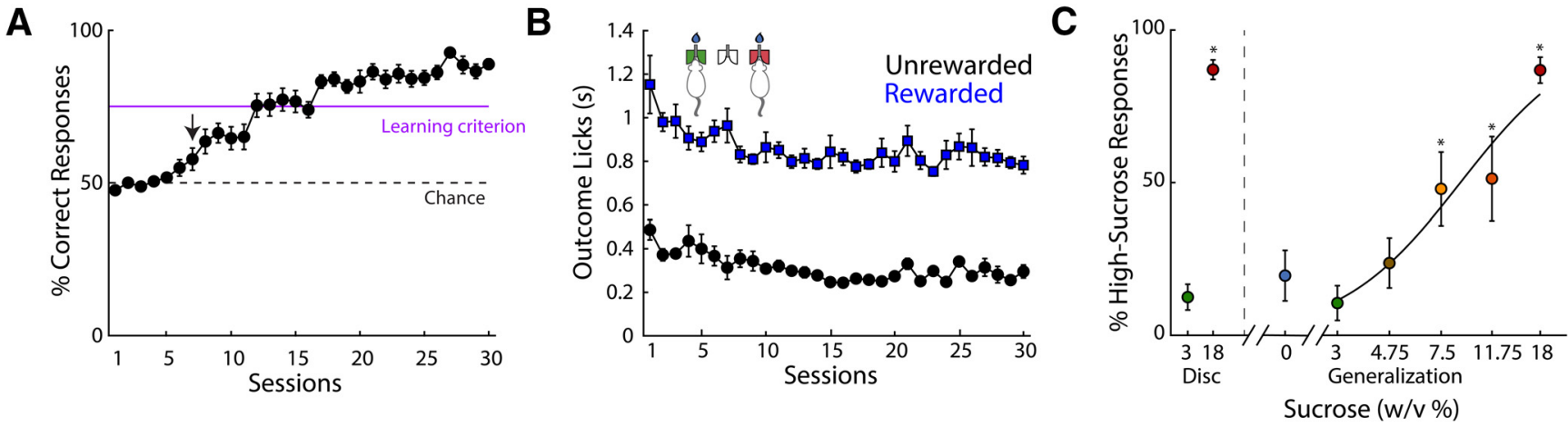

Figure 2. Subjects guide their decisions based on sucrose's intensity. A, Performance of subjects across discrimination (disk) sessions. After seven sessions, their performance in distinguishing $3 \%$ and $18 \%$ sucrose was above chance level (dashed line), while learning criterion (purple line; $\geq 75 \%$ correct responses during four consecutive sessions) was reached in $19 \pm 4$ sessions. The arrow at day 7 indicates that performance was significantly different compared with day 1 . B, Duration of lateral outcome epoch during rewarded (blue) and unrewarded (black) trials. From day 1, licking was significantly longer in rewarded than in unrewarded trials. $\boldsymbol{C}$, Percentage of responses where the subject perceived a sucrose concentration as high, during discrimination (disk) trials on the left side of the dashed line and generalization trials from the vertical gray dashed line to the right side. The $x$-axis is scaled logarithmically. * Significantly different from 3\%-discrimination trials. Data are presented as mean \pm SEM. For other behavioral measurements that were not significantly affected by the sucrose concentration, see Extended Data Figure 2-1.

(Fig. 1C). During generalization trials, the subjects were presented with water ( $0 \%$ sucrose) or one of five sucrose concentrations $(3 \%, 4.75 \%, 7.5 \%, 11.75 \%$, and $18 \%)$, which they had to classify as either "low" or "high." Figure $2 \mathrm{C}$ shows a plot of the perceived sucrose concentration as "high" as a function of sucrose concentration $\left(F_{(7,64)}=78.2\right.$; $p<0.0001)$. In comparison to the $3 \%$ sucrose-discrimination trials $(p<0.0001$; Fig. $2 C$, left side), subjects made significantly more "high" judgments of sucrose concentrations $\geq 7.5 \%$. In sum, as reported by Fonseca et al. (2018), the subjects could make perceptual decisions about sucrose's intensity. That said, we are aware of the possibility that animals could guide their decisions based on either sucrose's intensity or its palatability since for sucrose both attributes covary (St. John and Spector, 2008). To distinguish between these possibilities, using a CTA, we altered the palatability of sucrose from appetitive to aversive.

\section{A CTA to $18 \%$ sucrose changes its palatability from positive to negative}

In order to dissociate sucrose's perceived intensity attribute from its palatability, we changed the palatability component. This was achieved by inducing a CTA using $7.5 \mathrm{ml} / \mathrm{kg}$ of $0.2 \mathrm{~m} \mathrm{LiCl}$ (a visceral malaise agent) that was paired to the consumption of $18 \%$ sucrose. Figure $3 A$ depicts the experimental protocol. Briefly, after training the animals in the sucrose intensity task (Fig. 2), they then received a CTA to $18 \%$ sucrose (Fig. $3 B$ ). This was then followed by re-evaluation of the intensity task (Fig. 3C). Finally, a two-bottle preference test between water and sucrose $18 \%$ was given (see below). Because of the extremely rewarding effects of sucrose (la Fleur et al., 2007; Lenoir et al., 2007), we repeated the CTA procedure twice to achieve a longer-lasting aversion (Fig. $3 B$ ). Furthermore, given that the CTA is stronger for the specific concentration used as a conditioned stimulus
(Richardson et al., 1984), we paired LiCl against 18\% sucrose, which is the most salient stimulus in the task. We found that the amount of water ingested during the first $15 \mathrm{~min}$ in the Baseline (BL) epoch, as well as the intake of sucrose before the acquisition of CTA, was the same in rats injected intraperitoneally either with saline or LiCl (BL: $U=8, p>0.05$; sucrose: $U=7, p<0.05$ ). However, once a CTA was established, the animals injected with $\mathrm{LiCl}$ consumed a significantly lower volume of $18 \%$ sucrose $(5.0 \pm 2.5 \mathrm{ml})$ than the saline group $(21.5 \pm 2.0 \mathrm{ml} ; U=0 ; p<0.02$; see test $1+$ CTA-2 d). These differences were slightly larger on the second test day (test $2 ; U=0, p<0.01$ ). In sum, as expected, given a CTA successfully changed the hedonic value of $18 \%$ sucrose from appetitive to aversive.

\section{Changes in sucrose's palatability left intensity-guided perceptual judgments practically unaffected}

To determine whether the subjects could classify sucrose concentrations by their intensity after a CTA, we reevaluated their sucrose intensity task performance. Although we observed a significant decrease in the percent correct performance before and after the change in palatability from appetitive to aversive for the CTA group (from $92.1 \pm 0.8 \%$ to $89.2 \pm 0.9 \%$ correct from pre-CTA to post-CTA, $t_{(23)}=2.72 ; p<0.0001$; Table 1 ), all subjects continued performing well above the learning criterion after CTA (Extended Data Fig. 3-1). Moreover, the performance was not significantly different between saline post-CTA and LiCl post-CTA $\left(t_{(41)}=1.23\right.$; $\left.p>0.05\right)$, suggesting that changes in positive palatability did not affect the perceived intensity. Likewise, the performance preCTA versus post-CTA of the Saline group was unaffected $\left(t_{(16)}=0.89, p>0.05\right)$. Extended Data Figure 3-1 and Table 1 show a detailed description of other behavioral measurements in the discrimination sessions unaffected by the CTA procedure. Concerning this study, we found that 
A

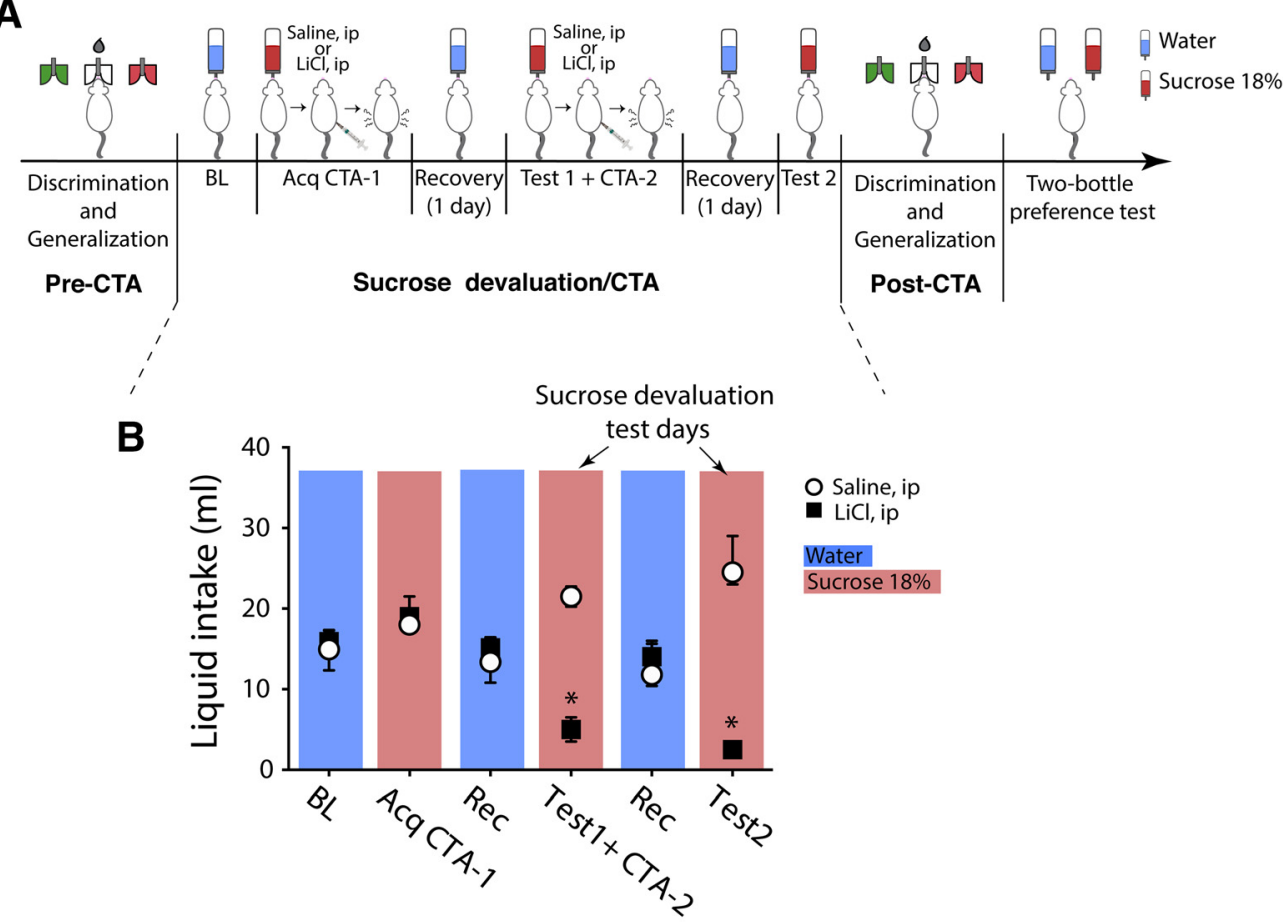

C

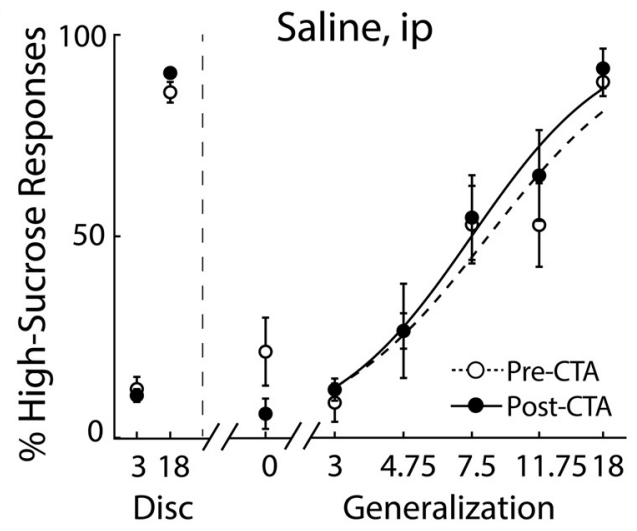

Sucrose (w/v \%)
D

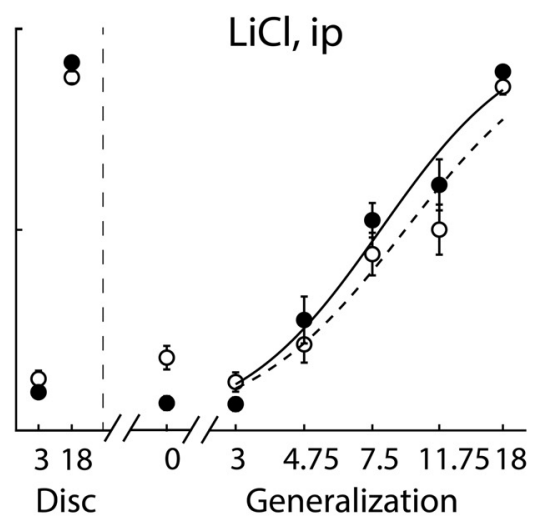

Sucrose $(w / v \%)$

Figure 3. Devaluating sucrose's hedonically positive palatability does not affect sucrose's response to concentration (intensity). $\boldsymbol{A}$, Timeline of the experimental design. After the animals were trained to identify sucrose concentrations, as shown in Figures 1, 2 (discrimination disk and generalization; pre-CTA), the subjects went through a sucrose devaluation procedure involving two consecutive CTAs. As a control, a baseline water consumption (blue bottle) was measured over $15 \mathrm{~min}$. The next day after baseline testing for water, the animals were given $18 \%$ sucrose (red bottle) to consume, and 15 min later, they received an intraperitoneal injection of $0.2 \mathrm{~m}$ lithium chloride ( $\mathrm{LiCl}$ ), a visceral malaise agent [Acquisition of first CTA (Acq CTA-1)]. Then, after a recovery day, the subjects were again given $18 \%$ sucrose for $15 \mathrm{~min}$ to consume followed by the second injection of $0.2 \mathrm{M} \mathrm{LiCl}$ or saline (test $1+\mathrm{CTA}-2$ ). After a recovery day, test 2 was performed by measuring the intake of $18 \%$ sucrose. After the CTA protocol, sucrose intensity task was re-evaluated (pre-CTA vs post-CTA, see $\boldsymbol{C}, \boldsymbol{D}$ ). Finally, a sucrose preference test was conducted using a two-bottle test to confirm that it remained aversive at the end of the experiments (for results, see Fig. 4). B, A histogram showing liquid intake for several of the epochs shown in dashed line expansion of $\boldsymbol{A} ; 15 \mathrm{~min}$ after liquid intake, rats were injected with saline (open circle) or $0.2 \mathrm{M}$ LiCl (black rectangles). Blue and red background shadows depict conditions where subjects had access to water and sucrose, respectively. The two upper arrows indicate the two test days where it can be seen that subjects developed CTA. * Significant differences between saline and $\mathrm{LiCl}$ groups. Data are shown as median \pm iqr. $\boldsymbol{C}$, Percentage of times the subject in the saline group classified a sucrose concentration as "high." Performances obtained before and after CTA are represented by the open, and black filled circles, respectively. The dashed (pre-CTA) and solid (post-CTA) lines are the fitted sigmoid, respectively. The $x$-axis is scaled logarithmically. Data are presented as mean \pm SEM. $\boldsymbol{D}$, Same as in $\boldsymbol{C}$ but for rats injected with LiCl. These results suggest that sucrose's intensity attribute is the main orosensory feature used by rats to solve the task. Extended Data Figure 3-1 shows values for the other behavioral measurements, and Extended Data Figure 3-2 shows the performance of each individual rat. 
Table 1: Behavioral changes in sucrose intensity task after CTA to $18 \mathrm{w} / \mathrm{v} \%$ sucrose

\begin{tabular}{|c|c|c|c|c|}
\hline \multirow[b]{2}{*}{ Behavioral measurements } & \multicolumn{2}{|c|}{ Saline, i.p. } & \multicolumn{2}{|c|}{ LiCl, i.p. } \\
\hline & Pre-CTA & Post-CTA & Pre-CTA & Post-CTA \\
\hline Performance (\%) & $87.7 \pm 3$ & $87.3 \pm 2.7$ & $92.1 \pm 0.8$ & $89.2 \pm 0.9 *$ \\
\hline CDL (s) & $0.5 \pm 0.1$ & $0.6 \pm 0.1$ & $0.6 \pm 0.04$ & $0.5 \pm 0.04$ \\
\hline Response (s) & $0.8 \pm 0.04$ & $0.9 \pm 0.04$ & $0.8 \pm 0.04$ & $0.8 \pm 0.05$ \\
\hline Return (s) & $1.8 \pm 0.05$ & $1.8 \pm 0.02$ & $1.9 \pm 0.06$ & $1.9 \pm 0.05$ \\
\hline Number of trials & $249 \pm 30.2$ & $258.5 \pm 13.7$ & $270.2 \pm 2.8$ & $258.7 \pm 8.6$ \\
\hline PSE & $9.6 \pm 0.6$ & $7.6 \pm 0.4 *$ & $9.4 \pm 3.3$ & $8.6 \pm 2.9$ \\
\hline $\mathrm{Li}$ & $4.7 \pm 0.4$ & $3.5 \pm 0.4$ & $3.3 \pm 0.5$ & $2.8 \pm 0.4$ \\
\hline WF & $0.50 \pm 0.05$ & $0.42 \pm 0.04$ & $0.36 \pm 0.05$ & $0.33 \pm 0.05$ \\
\hline
\end{tabular}

Data presented as mean \pm SEM. Data averaged from the five last and first discrimination (from performance to number of trials) and generalization (PSE, Li, WF) sessions, before and after CTA procedure. * Indicates significant difference. All statistical results can be found in the Extended Data Table 1-1.

the percentage of animals judging sucrose as "high" as a function of sucrose concentration was unchanged from before to after CTA, for the saline $\left(F_{(1,30)}=0.16, p>0.05\right.$; Fig. $3 C)$ and the $\mathrm{LiCl}\left(F_{(1,40)}=0.65, p>0.05\right.$; Fig. $\left.3 D\right)$ groups. Details of the PSE (that is, the concentration that it is equally likely to be perceived as "low" or "high"), Li (refers to the amount of concentration that needs to be changed to perceive that a change in the stimulus has occurred), and WF (a sensitivity measure) are found in Table 1. Briefly, only PSE decreased significantly for the saline $\left(t_{(19)}=2.85, p<0.05\right)$ but not for the $\mathrm{LiCl}\left(t_{(17)}=0.68, p<0.05\right)$ group. On the other hand, the Li (saline $t_{(15)}=1.42, p>0.05$; saline $t_{(15)}=0.85$, $p>0.05$ ) and the WF (saline $t_{(15)}=0.91, p>0.05$; saline $t_{(15)}$ $=0.47, p>0.05$ ) were not significantly affected by sucrose devaluation (Table 1; Extended Data Fig. 3-2). That is, the sensitivity to identify sucrose concentrations was unaffected by the CTA procedure. Therefore, to solve the task, subjects used the taste information contained in the perceived intensity attribute, which was essentially unaffected by changes in the hedonically positive component of palatability.

\section{The shift of $18 \%$ sucrose's valence from positive to negative persists after re-evaluation on the sucrose intensity task}

To demonstrate that in the sucrose intensity task, the rejection of sucrose remained until the end of re-evaluation, we performed a two-bottle preference test (water vs
$18 \%$ sucrose; Fig. 4A). A PR was calculated by dividing the sucrose intake amount by the total intake of sucrose + water. Note that PRs from 0 to 0.5 indicate water was preferred, and PRs from 0.5 to 1 means $18 \%$ sucrose was preferred. For the saline and $\mathrm{LiCl}$ groups, the difference in PR during both testing days was significantly different (day 1: $U=0, p<0.02$; day 2: $U=0, p<0.02$; Fig. $4 B$ ). The control saline group displayed a high-sucrose preference that was consistent across days (day 1: $0.92 \pm 0.06$; day $2: 0.97 \pm 0.04 ; W=10, p>0.05$ ). No subject showed sucrose preference for the $\mathrm{LiCl}$ group compared with the control group (Fig. 4B). The consumption pattern was similar during both test days (day 1: $0.27 \pm 0.45$; day 2: $0.27 \pm 0.14 ; W=9, p>0.05)$. Thus, $18 \%$ of sucrose remained aversive even after the subjects correctly classified sucrose concentrations during the re-evaluation test. That said, in this task, animals were using information about the concentration (intensity) rather than the hedonic palatability to make their taste perceptual decisions about sucrose's perceived intensity.

\section{Discussion}

The encoding of tastants consists of at least two processes that involve its quality and its palatability. The quality of sucrose is presumed to remain constant for all concentrations (Pfaffmann, 1941; Berridge and Grill, 1983; Richardson et al., 1984; Gutierrez et al., 2020;
A

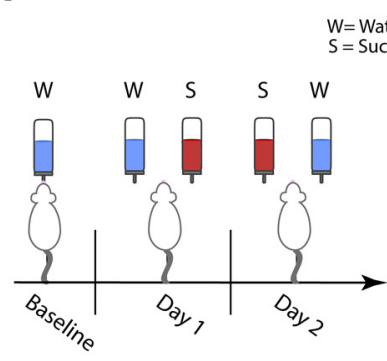

B

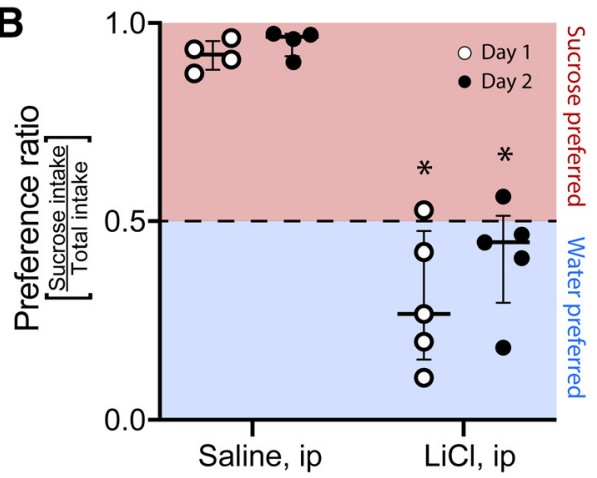

Figure 4. The CTA to $18 \%$ sucrose was not extinguished after re-evaluating performance in the sucrose intensity task (post-CTA; Fig. 3A). A, Two bottle experiment design. B, Sucrose PR for saline and LiCl groups during day 1 (white circles) and day 2 (black circles) of the two-bottle preference test. Note that, compared with saline treated rats, subjects injected with LiCl persistently disliked and rejected sucrose. * Significant differences compared with saline-treated rats. Data are presented as median \pm iqr. 
Staszko et al., 2020), whereas its palatability can change depending on a variety of factors including learning and the animal's internal states (Rolls, 2007; Levitan et al., 2019). In the primary GC, there is a disagreement about whether taste quality is encoded by narrowly-tuned and spatially confined neurons (Chen et al., 2011) or by both narrowly-tuned and broadly-tuned neurons distributed throughout the GC and other cortical regions (Jezzini et al., 2013; Fletcher et al., 2017; Levitan et al., 2019). Regarding palatability, there is also disagreement as to its encoding in the GC. One group of studies indicated that palatability occurs in a serial fashion after the quality and that it can even use the same neurons to encode both features (Katz et al., 2001; Jezzini et al., 2013; Vincis et al., 2020). In contrast, another study found that quality and palatability are processed by independent circuits, and that palatability can be changed without changing taste quality (Wang et al., 2018). There is, however, a third sensory attribute also encoded in the GC, namely, the perceived intensity of a tastant. It is presumed that as the concentration is increased (decreased), its perceived intensity will follow (Gautam et al., 2012; MacDonald et al., 2012; Blonde et al., 2015), assuming that the quality remains unchanged as is the case for sucrose. In this study, we developed a protocol in which animals tasting solutions of sucrose reported their perceived intensity. Then, using a CTA to change sucrose's palatability from hedonically positive to negative, we found that the perceived intensity remained unchanged, showing that these responses were primarily guided by its intensity and not by its palatability attribute. This indicates that the hedonic aspects of palatability, which are associated with the oromotor aspects of eating (Berridge and Grill, 1983), are not necessary for the animals to perceive the intensity of a tastant.

\section{Taste quality and palatability}

Stimulating the tongue with sugars activates "sweet" taste receptor cells (TRCs and their associated brain circuits; Huang et al., 2006) that are then translated into multiple attribute representations informing the rodents about what we assume is similar to what humans experience as sweetness (Breslin, 2013; Wang et al., 2018; Gutierrez et al., 2020). Beyond expectation, this process is initiated with the presence of a chemical stimulus in the mouth and then with the formation of its quality and palatability attributes. Data in rodents currently indicate that these attributes could be formed either serially and/or in parallel. The serial model is supported by electrophysiological recordings (Katz et al., 2001; Levitan et al., 2019; Vincis et al., 2020) and suggests that these attributes are formed temporally with quality giving rise to palatability. Alternatively, one could also argue that a dynamic network of parallel circuits could provide information to GC differently over time. In this regard, the study by Samuelsen et al. (2013) found that pharmacological inactivation of ventroposteromedial nucleus of the thalamus (VPMpc; i.e., the primary gustatory thalamus) disrupted taste coding in a subpopulation of GC neurons although it was not completely eliminated. These findings support a parallel and distributed processing mechanism for inputs converging onto the GC. Moreover, the parallel processing model is also supported by the work on decerebrate rats (Grill and Norgren, 1978a). These animals were decerebrated at the level of the superior colliculus and thus do not have intact connections to the VPMpc that, in turn, projects to the GC, an area that plays a key role in gustatory processing, taste-guided decisions, taste-aversion learning, taste sensitivity and taste recognition (Yamamoto et al., 1980; Cechetto and Saper, 1987; Cubero et al., 1999; Kobayashi, 2011; Samuelsen et al., 2013; Blonde et al., 2015; Peng et al., 2015; Fletcher et al., 2017; Fonseca et al., 2018). Nevertheless, these animals still give normal hedonically positive oral responses to sucrose (Grill and Norgren, 1978a), indicating that palatability alone can drive basic oromotor reflexes elicited by sucrose. Although the forebrain is required to update new associative changes in palatability, it is important to note that in decerebrate rats, a CTA cannot be conditioned (Grill and Norgren, 1978a). Other evidence supporting, but not proving, that taste quality and palatability could be processed by different brain circuits comes from studies using TRPM5 knock-out mice. These mice are partially "blind" to compounds that humans perceive as sweet (Banik et al., 2018). They have a diminished ability to detect sucrose but could still learn a reduced, but selective, CTA to sucrose (Eddy et al., 2012). Interestingly, they showed a normal ability to develop a conditioned flavor aversion to a sweet and odor mixture, suggesting that these mice maintained the ability to update the palatability despite the impairment in taste detection (Eddy et al., 2012). Although these results have been interpreted as evidence for TRPM5-independent pathway to detect sweet tastants (Eddy et al., 2012; see also Banik et al., 2018), it could be argued that it also supports the existence of independent-parallel processing of palatability and taste quality. In this parallel model, after sugar activates the sweet responsive TRCs, the identity (quality) and the palatability should arise more or less in parallel, and no assumption is made that one attribute drives the other. Whichever hypothesis proves to be correct, it is clear that once taste attributes are formed, they then became independent because, as previously noted, sweet taste quality recognition can be dissociated from its palatability (Wang et al., 2018). Here, we extend these observations by demonstrating that sucrose's perceived intensity and hedonically positive palatability can also be dissociated at the behavioral level.

\section{Dissociation of taste intensity from palatability}

A third sensory attribute conveying information about a tastant is its intensity (Lawless and Skinner, 1979). In the periphery, increasing the concentration should activate more receptors and thus increase the intensity (Beidler, 1954; Wu et al., 2015). However, from a behavioral viewpoint, although related (Lawless and Skinner, 1979), the perceived intensity is not reflected by any licking responses per se, but rather it is a process that requires the animal to make a decision regarding whether one concentration of a tastant is higher or lower than a standard concentration. Using a sucrose intensity task combined with CTA, we found behavioral evidence supporting that the perceived taste intensity could be separately processed 
from the hedonically positive part of sucrose's palatability (Fig. 3).

Although the reason(s) the gustatory system seems to process palatability distinctly from intensity is not known, one possibility is that it could result in a sharpening of aversions to a specific tastant and its concentration, rather than generalizing them to an entire class of foods with similar features. In this regard, intrachemical and interchemical CTA generalization studies had found that for a particular tastant, rats develop the strongest aversion to a given tastant concentration when it is paired with visceral malaise and exhibit smaller aversions to either lower or higher concentrations of the same tastant (Tapper and Halpern, 1968; Rozin et al., 1979; Richardson et al., 1984; Eddy et al., 2012). We argue that the same principle (i.e., the concentration of sucrose can be a cue) is used by rats to solve our sucrose intensity task because they can use $10-\mu l$ drops of $18 \%$ sucrose (maximum tested concentration) as a cue to classify it as high and to obtain a water reward, regardless of whether it was paired with malaise. Alternatively, and given that it is expected that the CTA to $18 \%$ sucrose would have a smaller effect on changing the palatability of lower $3 \%$ sucrose concentration (Richardson et al., 1984). In this regard, one possibility is that after CTA the rats guided their choices based solely on the intensity of the lower $3 \%$ concentration. However, we found this possibility to be improbable since they judged the intensity of $3 \%$ sucrose as "low" and the 18\% sucrose as "high" in a comparable manner before and after CTA (Fig. 3C). The well-organized increase in high responses as a function of sucrose concentration suggests that concentration exerted and maintained stimulus control over the behavior before and after CTA. Thus, we conclude that the most parsimonious explanation of our results is that the hedonically positive palatability of sucrose is not needed to identify its intensity.

\section{Dissociation of taste quality from the intensity}

To our knowledge, no attempt has been made to dissociate taste quality from the perceived intensity, although Gautam et al. (2012) found that intensity is the most crucial dimension to explain taste quality. However, in humans, neither taste adaptation nor cross-adaptation procedures produced changes in tastant quality (Meiselman, 1968). For example, after overstimulation with a high-sucrose concentration, it is possible to experience as less intense a lower sucrose concentration without affecting its sweet quality (Meiselman, 1968). This suggests that quality and intensity could also be separately processed, as suggested by our behavioral experiments for palatability. That is, the hedonically positive palatability responses evoked by sucrose are not necessary for animals to detect its perceived intensity.

\section{References}

Bagiella E, Sloan RP, Heitjan DF (2000) Mixed-effects models in psychophysiology. Psychophysiology 37:13-20.

Banik DD, Martin LE, Freichel M, Torregrossa A-M, Medler KF (2018) TRPM4 and TRPM5 are both required for normal signaling in taste receptor cells. Proc Natl Acad Sci USA 115:E772-E781.

Beidler LM (1954) A theory of taste stimulation. J Gen Physiol 38:133-139.
Berridge KC, Grill HJ (1983) Alternating ingestive and aversive consummatory responses suggest a two-dimensional analysis of palatability in rats. Behav Neurosci 97:563-573.

Berridge KC, Robinson TE, Aldridge JW (2009) Dissecting components of reward: 'liking', 'wanting', and learning. Curr Opin Pharmacol Neurosci 9:65-73.

Blonde GD, Bales MB, Spector AC (2015) Extensive lesions in rat insular cortex significantly disrupt taste sensitivity to $\mathrm{NaCl}$ and $\mathrm{KCl}$ and slow salt discrimination learning. PLoS One 10:e0117515.

Breslin PAS (2013) An evolutionary perspective on food and human taste. Curr Biol 23:R409-R418.

Cechetto DF, Saper CB (1987) Evidence for a viscerotopic sensory representation in the cortex and thalamus in the rat. $\mathrm{J}$ Comp Neurol 262:27-45.

Chen X, Gabitto M, Peng Y, Ryba NJP, Zuker CS (2011) A gustotopic map of taste qualities in the mammalian brain. Science 333:12621266.

Church RM, Deluty MZ (1977) Bisection of temporal intervals. J Exp Psychol Anim Behav Process 3:216-228.

Colbert CL, Garcea M, Spector AC (2004) Effects of selective lingual gustatory deafferentation on suprathreshold taste intensity discrimination of $\mathrm{NaCl}$ in rats. Behav Neurosci 118:1409-1417.

Cubero I, Thiele TE, Bernstein IL (1999) Insular cortex lesions and taste aversion learning: effects of conditioning method and timing of lesion. Brain Res 839:323-330.

Davis JD (1973) The effectiveness of some sugars in stimulating licking behavior in the rat. Physiol Behav 11:39-45.

Dolensek N, Gehrlach DA, Klein AS, Gogolla N (2020) Facial expressions of emotion states and their neuronal correlates in mice. Science 368:89-94.

Domjan M (1975) The nature of the thirst stimulus: a factor in conditioned taste-aversion behavior. Physiol Behav 14:809-813.

Dotson CD, Spector AC (2004) The relative affective potency of glycine, I-serine and sucrose as assessed by a brief-access taste test in inbred strains of mice. Chem Senses 29:489-498.

Dunnett CW (1955) A multiple comparison procedure for comparing several treatments with a control. J Am Stat Assoc 50:1096-1121.

Eddy MC, Eschle BK, Peterson D, Lauras N, Margolskee RF, Delay ER (2012) A conditioned aversion study of sucrose and SC45647 taste in TRPM5 knockout mice. Chem Senses 37:391-401.

Fletcher ML, Ogg MC, Lu L, Ogg RJ, Boughter JD (2017) Overlapping representation of primary tastes in a defined region of the gustatory cortex. J Neurosci 37:7595-7605.

Fonseca E, de Lafuente V, Simon SA, Gutierrez R (2018) Sucrose intensity coding and decision-making in rat gustatory cortices. Elife 7:e41152.

Garcia J, Lasiter PS, Bermudez-Rattoni F, Deems DA (1985) A general theory of aversion learning. Ann NY Acad Sci 443:8-21.

Gautam SH, Rebello MR, Verhagen JV (2012) Taste quality and intensity of 100 stimuli as reported by rats: the taste-location association task. Front Behav Neurosci 6:19.

Grill HJ, Norgren R (1978a) Chronically decerebrate rats demonstrate satiation but not bait shyness. Science 201:267-269.

Grill HJ, Norgren R (1978b) The taste reactivity test. I. Mimetic responses to gustatory stimuli in neurologically normal rats. Brain Res 143:263-279.

Grill HJ, Norgren R (1978c) The taste reactivity test. II. Mimetic responses to gustatory stimuli in chronic thalamic and chronic decerebrate rats. Brain Res 143:281-297.

Gutierrez R, Fonseca E, Simon SA (2020) The neuroscience of sugars in taste, gut-reward, feeding circuits, and obesity. Cell Mol Life Sci 77:3469-3502.

Huang AL, Chen X, Hoon MA, Chandrashekar J, Guo W, Tränkner D, Ryba NJP, Zuker CS (2006) The cells and logic for mammalian sour taste detection. Nature 442:934-938.

Jezzini A, Mazzucato L, La Camera G, Fontanini A (2013) Processing of hedonic and chemosensory features of taste in medial prefrontal and insular networks. J Neurosci 33:18966-18978. 
Katz DB, Simon SA, Nicolelis MAL (2001) Dynamic and multimodal responses of gustatory cortical neurons in awake rats. J Neurosci 21:4478-4489.

Kerby DS (2014) The simple difference formula: an approach to teaching nonparametric correlation. Compr Psychol 3:11.IT.3.1.

Kobayashi M (2011) Macroscopic connection of rat insular cortex: anatomical bases underlying its physiological functions. Int Rev Neurobiol 97:285-303.

Kotlus BS, Blizard DA (1998) Measuring gustatory variation in mice: a short-term fluid-intake test. Physiol Behav 64:37-47.

la Fleur SE, Vanderschuren LJMJ, Luijendijk MC, Kloeze BM, Tiesjema B, Adan RA (2007) A reciprocal interaction between food-motivated behavior and diet-induced obesity. Int $\mathrm{J}$ Obes (Lond) 31:1286-1294.

Lawless HT, Skinner EZ (1979) The duration and perceived intensity of sucrose taste. Percept Psychophys 25:180-184.

Lenoir M, Serre F, Cantin L, Ahmed SH (2007) Intense sweetness surpasses cocaine reward. PLoS One 2:e698.

Levitan D, Lin JY, Wachutka J, Mukherjee N, Nelson SB, Katz DB (2019) Single and population coding of taste in the gustatory cortex of awake mice. J Neurophysiol 122:1342-1356.

MacDonald CJ, Meck WH, Simon SA (2012) Distinct neural ensembles in the rat gustatory cortex encode salt and water tastes. J Physiol 590:3169-3184.

Meiselman HL (1968) Adaptation and cross-adaptation of the four gustatory qualities. Percept Psychophys 4:368-372.

Ninomiya $\mathrm{Y}$, Higashi T, Katsukawa H, Mizukoshi T, Funakoshi M (1984) Qualitative discrimination of gustatory stimuli in three different strains of mice. Brain Res 322:83-92.

Peng Y, Gillis-Smith S, Jin H, Tränkner D, Ryba NJP, Zuker CS (2015) Sweet and bitter taste in the brain of awake behaving animals. Nature 527:512-515.

Perez IO, Villavicencio M, Simon SA, Gutierrez R (2013) Speed and accuracy of taste identification and palatability: impact of learning, reward expectancy, and consummatory licking. Am J Physiol Regul Integr Comp Physiol 305:R252-R270.

Pfaffmann C (1941) Gustatory afferent impulses. J Cell Comp Physiol $17: 243-258$
Richardson R, Williams C, Riccio DC (1984) Stimulus generalization of conditioned taste aversion in rats. Behav Neural Biol 41:41-53.

Rolls ET (2007) Sensory processing in the brain related to the control of food intake. Proc Nutr Soc 66:96-112.

Rozin P, Gruss L, Berk G (1979) Reversal of innate aversions: attempts to induce a preference for chili peppers in rats. J Comp Physiol Psychol 93:1001-1014.

Samuelsen CL, Gardner MPH, Fontanini A (2013) Thalamic contribution to cortical processing of taste and expectation. J Neurosci 33:1815-1827.

Spector AC, Smith JC (1984) A detailed analysis of sucrose drinking in the rat. Physiol Behav 33:127-136.

Spector AC, Klumpp PA, Kaplan JM (1998) Analytical issues in the evaluation of food deprivation and sucrose concentration effects on the microstructure of licking behavior in the rat. Behav Neurosci 112:678-694.

St. John SJ, Spector AC (2008) Behavioral analysis of taste function in rodent models. In: The senses: a comprehensive reference (Masland RH, Albright TD, Dallos P, Oertel D, Firestein S, Beauchamp GK, Bushnell MC, Basbaum AI, Kaas JH, Gardner EP, eds), pp 409-427. New York: Academic Press.

Staszko SM, John D, Boughter J, Fletcher ML (2020) Taste coding strategies in insular cortex. Exp Biol Med (Maywood) 245:448455.

Tapper DN, Halpern BP (1968) Taste stimuli: a behavioral categorization. Science 161:708-710.

Vincis R, Chen K, Czarnecki L, Chen J, Fontanini A (2020) Dynamic representation of taste-related decisions in the gustatory insular cortex of mice. Curr Biol 30:1834-1844.e5.

Wang L, Gillis-Smith S, Peng Y, Zhang J, Chen X, Daniel Salzman C, Ryba NJP, Zuker CS (2018) The coding of valence and identity in the mammalian taste system. Nature 558:127-131.

Wu A, Dvoryanchikov G, Pereira E, Chaudhari N, Roper SD (2015) Breadth of tuning in taste afferent neurons varies with stimulus strength. Nat Commun 6:8171.

Yamamoto T, Matsuo R, Kawamura Y (1980) Localization of cortical gustatory area in rats and its role in taste discrimination. J Neurophysiol 44:440-455. 